\title{
Enjeux du dialogue et écriture polyphonique dans le "Supplément au Voyage de Bougainville"
}

\section{Pierino Gallo}

\section{(2) OpenEdition}

\section{Journals}

\section{Édition électronique}

URL : http://journals.openedition.org/studifrancesi/14591

DOI : 10.4000/studifrancesi. 14591

ISSN : 2421-5856

\section{Éditeur}

Rosenberg \& Sellier

\section{Édition imprimée}

Date de publication : 1 décembre 2018

Pagination : 425-432

ISSN : 0039-2944

\section{Référence électronique}

Pierino Gallo, «Enjeux du dialogue et écriture polyphonique dans le "Supplément au Voyage de Bougainville" ", Studi Francesi [En ligne], 186 (LXII | III) | 2018, mis en ligne le 01 décembre 2019, consulté le 05 janvier 2021. URL : http://journals.openedition.org/studifrancesi/14591 ; DOI : https:// doi.org/10.4000/studifrancesi.14591

\section{(c) (i) $\odot$}

Studi Francesi è distribuita con Licenza Creative Commons Attribuzione - Non commerciale - Non opere derivate 4.0 Internazionale. 


\title{
Enjeux du dialogue et écriture polyphonique dans le "Supplément au Voyage de Bougainville"
}

\begin{abstract}
Written in 1772 and first presented to the public in 1796, Diderot's Supplément au Voyage de Bougainville occupies a prominent place in the history of primitivism. Our study aims to show how, in this work, the processes of creation - embedded narratives, speeches and the polyphonic structure of the text - set up an ontological mechanism including an image of the "Other" and the "Self" which deserves to be analysed. Far from being resolved, the opposition between these two poles draws a divided subject which is made in language and manifests itself in and through a complex network of voices whose functioning is neither identical nor even complementary. It is by this movement, made up of aporias and paradoxes, that dialogical practice acquires in Diderot its full originality, becoming both a method of interpretation and the product of this interpretation.
\end{abstract}

Lorsqu'on s'interroge sur la place et la signification que la forme dialoguée peut avoir au XVIII siècle, ces mots tirés de la pensée philosophique 29 semblent s'imposer comme une évidence: «On doit exiger de moi que je cherche la vérité, mais non que je la trouve» ${ }^{1}$. Ce passage, symptomatique de la démarche déployée par Diderot dans presque tous ses écrits, contribue à asseoir la réputation du philosophe en tant que divulgateur de la «pensée questionneuse» et à faire de lui un défenseur du dialogue ${ }^{2}$. Jamais, peut-être, la conscience d'un auteur n'a trouvé à se mouler aussi parfaitement dans une forme littéraire. Et pourtant, sa pratique dialogique a quelque chose de nouveau: dénuée de son but didactique, elle incarne un terrain où des idées se heurtent, des questions et des réponses se succèdent en suscitant à leur tour d'autres interrogations. Rejetant tout esprit de système, «Diderot nous livre une pensée qui se fait devant nous, qui se cherche en tâtonnant, qui s'affronte à lui, mais aussi à elle-même» ${ }^{3}$. C'est assez dire l'écart qui le sépare de l'ancienne maïeutique ${ }^{4}$, vivifiée par les théories de Marmontel $^{5}$, ou encore de l'heuristique platonicienne, basée sur des schémas linéaires.

(1) D. Diderot, Pensées philosophiques, dans Euvres philosophiques, éd. M. Delon, Paris, Gallimard, 2010, «Bibliothèque de la Pléiade», p. 16.

(2) «With no other author did the dialogue so thoroughly penetrate all levels of thought and work» (C. Sherman, Diderot and the Art of Dialogue, Genève, Droz, 1976, p. 12).

(3) R. Mortier, Diderot et le problème de l'expressivité: de la pensée au dialogue heuristique, «Cahiers de l'Association internationale des études françaises»13, 1961, p. 285.

(4) «Pour moi qui m’occupe plutôt à former des nuages qu'à les dissiper, et à suspendre les jugements qu'à juger, je vais vous démontrer encore que si le paradoxe que je viens d'avancer n'est pas vrai, si nous n'avons pas plusieurs perceptions à la fois; il est impossible de raisonner et de discourir» (Lettre sur les sourds et muets, dans (Euvres philosophiques cit., pp. 222-223).

(5) Marmontel est l'auteur, avec l'abbé Morellet, de l'article «Dialogue» de l'Encyclopédie (IV, 936937). Ce texte, d'abord repris et remanié dans un article du Supplément (II), sera reproduit quelques ans plus tard dans les Éléments de littérature. Sa définition du «dialogue philosophique» doit beaucoup au modèle socratique: «Le difficile, je le répète, c'est de démêler, de classer, de circonscrire nos idées, en leur donnant toute leur étendue, d'en saisir les justes rapports, de tirer ainsi du chaos les éléments de la science et d'y répandre la lumière. C'est à quoi le dialogue philosophique est utilement employé parce qu'à mesure qu'il forme des nuages, il les dissipe; qu'à chaque pas il ne présente une nouvelle difficulté qu'afin de l'apla- 
Par son statut dynamique, le dialogue diderotien permet de jeter un pont entre stratégies d'écriture et postulations idéologiques, en recourant à une mise en scène complexe qui relève tant de l'histoire des idées que de l'analyse textuelle. Cette double oscillation, d'ailleurs, répond, chez notre auteur, à un cadre plus vaste: sa conception de la «multiplicité de l'homme, de l'être et de la vérité»'.

Ainsi, ce n'est pas un hasard si les années où cette réflexion atteint son apogée sont aussi, pour Diderot, celles des grandes narrations polyphoniques: à partir du Neveu de Rameau, rédigé en 1761, ce n'est qu'une suite d'entretiens et de commentaires $^{7}$, une chaîne de textes «ouverts» ${ }^{8}$ où l'aventure de la création va de pair avec une refondation de l'être humain.

Le Supplément au Voyage de Bougainville forme en ce sens un ensemble exemplaire. Texte hybride et fragmentaire, encadré par des dialogues-commentaires qui lui servent d'introduction et de conclusion, il enchaîne en quelques pages plusieurs actes de parole: un discours oratoire et un entretien dans la meilleure tradition primitiviste constituent son noyau'. A, B, le vieux Tahitien, Orou et l'Aumônier sont les acteurs de ce récit choral. On y devine, en lisant, un montage suspendu entre le vrai et le fictif, une mosaïque où se rencontrent plusieurs plans énonciatifs, derrière lesquels le philosophe se fait entendre: «une critique virulente des codes civil et religieux de l'Europe du XVIII ${ }^{\mathrm{e}}$ siècle s'y articule avec un rêve tahitien, véhiculé par un dialogue protéiforme dont les structures reflètent la complexité des enjeux autant que l'ambiguïé du propos» ${ }^{10}$. Or, de toutes les questions véhiculées par la forme, celles qui se rapportent à l'identité et à l'altérité se trouvent en tête de liste. D'abord, parce que l'Autre, implicite dans tout discours primitiviste, devient ici un sujet parlant, une condition sine qua non pour établir une confrontation efficace (aucun échange n'est possible en son absence ${ }^{11}$ ); ensuite, parce qu'il permet de comprendre, selon un jeu de miroir rendu possible par le dialogue, les diverses interprétations de l'œuvre sur la nature humaine, les mœurs et la civilisation.

Le but de notre étude est de montrer comment, dans le Supplément, les procédés de création - les récits enchâssés, les prises de parole et la structure polyphonique propre à ce texte - mettent en place un jeu subtil à valeur ontologique où se détache une image de l'Autre et du Moi en gestation. Bien loin d'être réglée, l'opposition de ces deux pôles dessine un sujet divisé qui se fait dans le langage et «se manifeste dans et par un réseau complexe de voix dont le fonctionnement n'est ni identique ni même

nir lui-même, et que son but est la solution de toutes celles que l'ignorance, l'habitude, l'opinion opposent à la vérité» (Éléments de littérature, éd. S. Le Ménahèze, Paris, Desjonquères, 2005, p. 401).

(6) R. Mortier, Diderot et le problème de l'expressivité cit., p. 284.

(7) Rappelons, par exemple, Le Rêve de d'Alembert (1769), l'Entretien d'un père avec ses enfants (1771), le triptyque dialogique formé par Ceci n'est pas un conte, Madame de La Carlière et le Supplément au Voyage de Bougainville (parus entre 1773 et 1774), ou encore l'Entretien d'un philosophe avec la maréchale de $* * * *$ (1776). Sans compter le statut fragmentaire de certains textes destinés à la Correspondance de Grimm ou à l'Histoire des deux Indes de l'abbé Raynal.

(8) «Par leurs formes autant que par leurs contenus, ces œuvres sont 'ouvertes' [...]. Ouvertes, parce que le texte clos prétend formellement à la totalité et à l'exhaustivité alors que la connaissance est par sa définition infinie et inachevée. Ouvertes, encore, parce qu'elles laissent place à la contradiction et à l'ambiguïté que la tradition cartésienne récuse fermement» (S. Pujol, Le Dialogue d'idées au dix-buitième siècle, Oxford, Voltaire Foundation, 2005, p. 309).

(9) Pour une étude plus étendue de la structure de l'œuvre, voir H. Dieckmann, Introduction, dans D. Diderot, Supplément au Voyage de Bougainville, Genève-Lille, Droz-Giard, 1955, p. XI-CLV.

(10) M. Mat, Le "Supplément au Voyage de Bougainville": une aporie polyphonique, «Revue internationale de philosophie»148-149, 1984, p. 159.

(11) Nous trouvons là le fondement de la «subjectivité» dont parle Benveniste: «La conscience de soi n'est possible que si elle s'éprouve par contraste. [...] Le langage n'est possible que parce que chaque locuteur se pose comme sujet, en renvoyant à lui-même comme je dans le discours. De ce fait, je pose une autre personne, celle qui, tout extérieure qu'elle est à "moi”, devient mon écho auquel je dis tu et qui me dit tu» (É. Benveniste, Problèmes de linguistique générale, I, Paris, Gallimard, 1966, p. 260). 
complémentaire» ${ }^{12}$. C'est par ce mouvement, fait d'apories et de paradoxes, que la pratique dialogique acquiert chez Diderot sa pleine fécondité, devenant à la fois une méthode d'interprétation et le produit de cette interprétation. Partant d'une analyse de l'écriture, on étudiera au même temps la manière dont le discours relie, non sans tensions, les concepts d'identité et d'altérité à d'autres axes thématiques tout au long des cinq parties du livre ${ }^{13}$. En ce sillage, une place importante sera accordée aux réflexions métalinguistiques, indices d'une «rhétorique signifiante». Cela, enfin, nous permettra de mieux situer la poétique diderotienne dans un projet plus vaste, à la fois esthétique et philosophique: celui d'une contre-narration de l'homme et du monde omniprésente chez les grandes figures du siècle.

Mais quelle est la substance du Supplément? Comment le philosophe la présentet-il aux lecteurs? À cet égard, le sous-titre fournit déjà d'utiles indications: Dialogue entre $A$ et $B$ sur l'inconvénient d'attacher des idées morales à certaines actions physiques qui n'en comportent pas ${ }^{14}$. Il s'agit, en résumant, d'un débat sur le rapport entre eros et morale. Le prétexte est fourni par le Voyage autour du monde de Louis-Antoine de Bougainville $^{15}$, et surtout par ses peintures de l'île de Tahiti. Séduit par cet ouvrage, Diderot en conçoit d'abord un compte rendu ${ }^{16}$, puis une suite imaginaire où, à travers un ailleurs réinventé, il engage une réflexion sur les rapports entre société et sexualité.

Le dialogue initial figure déjà, à ce propos, un moment de tension: appelés à encadrer le récit du Sauvage, deux locuteurs européens partent de Bougainville pour revenir encore une fois sur le binôme nature-société. Loin de proposer un modèle définitif, le discours débouche ici sur une pensée relativiste. La parabole d'Aotourou - le Tahitien que Bougainville amena en France - en est le point de départ:

A: Ô Aotourou, que tu seras content de revoir ton père, ta mère, tes frères, tes sœurs, tes compatriotes! Que leur diras-tu de nous?

B: Peu de choses et qu'ils ne croiront pas.

A: Pourquoi peu de choses?

B: Parce qu'il en a peu conçues, et qu'il ne trouvera dans sa langue aucuns termes correspondants à celles dont il a quelques idées.

\section{Et B de conclure:}

La vie sauvage est si simple, et nos sociétés sont des machines si compliquées! L'Otaïtien touche à l'origine du monde et l'Européen touche à sa vieillesse. L'intervalle qui le sépare de nous est plus grand que la distance de l'enfant qui naît à l'homme décrépit. (Supplément, p. 546)

On dirait, effectivement, une séquence dramatique ouverte, où la notion d'altérité est rattachée à une critique empirique du langage: la réalité n’est pas la même pour

(12) C.V. McDonald, Le Dialogue, l'utopie: "Le Supplément au voyage de Bougainville" par Denis Diderot, «Canadian Review of Comparative Literature / Revue Canadienne de Littérature Comparée» 3, Hiver 1976 , p. 67.

(13) «Jugement du Voyage de Bougainville» (I); «Les Adieux du Vieillard» (II); «L'Entretien de l'aumônier et d'Orou» (III-IV); «Suite du dialogue entre A et B» (V).

(14) Nos citations du Supplément seront issues de l'édition de Stéphane Pujol, dans Contes et romans, dir. M. Delon, Paris, Gallimard, 2004, «Bibliothèque de la Pléiade».

(15) Voyage autour du monde, par la frégate du roi «La Boudeuse», et la flûte «L'Étoile»; en 1766, 1767, 1768, et 1769, Paris, 1771.

(16) Le texte du Supplément ne devait être, au début, qu'un compte rendu destiné à la Correspondance littéraire. Malgré son amitié avec Grimm, Diderot ne parvint pas à le faire publier. 
tous, les références mentales diffèrent d'un lieu à l'autre. Les sections qui suivent, liées au «cadre» de façon dialectique, traduisent cette tension sur le mode de la fiction. Dans «Les Adieux du Vieillard», par exemple, l'Autre représenté est à la fois un sujet linguistique et social. La harangue met à nu une double violation: non seulement les Français ont «infecté» les mœurs des insulaires (en y introduisant la honte, les remords et l'effroi), mais ils en ont également corrompu le langage, leur code de lecture du réel. Ce morceau, au demeurant, est le produit d'une double traduction. Le commentaire est confié encore une fois à $\mathrm{A}$ et $\mathrm{B}$ :

A: Ce discours me paraît véhément, mais à travers je ne sais quoi d'abrupt et de sauvage il me semble retrouver des idées et des tournures européennes.

B: Pensez donc que c'est une traduction de l'otaïtien en espagnol et de l'espagnol en français. L'Otaïtien s'était rendu la nuit chez cet Ourou qu'il a interpellé et dans la case duquel l'usage de la langue espagnole s'était conservé de temps immémorial. Orou avait écrit en espagnol la harangue du vieillard, et Bougainville en avait une copie à la main, tandis que l'Otaïtien la prononçait. (Supplément, p. 551)

Ainsi conçue, donc, la mise en scène de l'Autre devient inséparable de la voix des narrateurs: sans leur parole, elle resterait incomprise, voire condamnée au silence. La structure narrative (il s'agit d'un discours rapporté) le confirme; l'acte d'énonciation du vieillard dépend d'un «locuteur primaire» qui est soit B soit l'auteur du Supplément.

Le même principe d'enchâssement revient dans «l'Entretien de l'aumônier et d'Orou». Ici, c'est le thème de la liberté entre les sexes qui engage Diderot dans une confrontation entre morale chrétienne et morale naturelle. Il n'est pas difficile de noter les stratégies textuelles cachées sous ce dialogue. Déjà au niveau quantitatif, les répliques du Tahitien sont plus longues et plus articulées que celles du religieux. La parole du Sauvage regagne ici sa véritable autorité, ses tirades développent une critique assez commune au siècle des Lumières. Invité à prendre une femme pour la nuit, l'Aumônier a beau opposer aux mœurs tahitiennes son «état» et sa «religion». Ses répliques se raccourcissent, se font plus fragmentaires au fur et à mesure que la nature triomphe.

Orou, qui a d'abord écouté ses raisons, lance une attaque à la doxa dominante. Une lecture attentive permet de distinguer dans son discours trois arguments principaux: le mariage tel que le peint l'Aumônier, est contraire à la nature, car tout lien permanent entre deux personnes fait de l'être pensant un objet de propriété. Deuxièmement, par son statut immuable, le mariage viole la loi générale de la nature, où tout être est dans un flux constant. Troisièmement, les autorités de l'Européen (Dieu, le prêtre et le magistrat) sont subjectives, donc arbitraires. Qui plus est, elles pourraient se trouver en désaccord et engendrer le désordre. En découle, chez l'homme civilisé, un conflit intérieur que reflètent, sur le plan énonciatif, les questions de l'Aumônier. On serait tenté d'y voir un des traits caractéristiques du dialogisme diderotien: «Avec Diderot, la quête se situe à proximité de la conscience. Grâce à cette problématisation réflexive du drame de l'énonciation, la démarche heuristique acquiert un contenu et une forme spécifiques» ${ }^{17}$. S'amorce ici une philosophie humaniste qui reviendra tout au long du Supplément: ce n'est qu'en écoutant la nature, qu'en reconnaissant l'altérité en lui-même, que le Français, «ni bon ni mauvais originellement», pourra résoudre ses conflits. «La grande entreprise - précise Gilbert Chinard - sera donc,

(17) R. BRIKI, Le dialogue romanesque au XVIII siècle, Tunis, Éditions Sahar, 2007, pp. 280-281. Voir aussi à ce sujet Y. Belaval, Études sur Diderot, Paris, Puf, 2003, p. 312. 
après nous être libérés des anciens préjugés, d'obtenir avant tout une connaissance exacte de la nature de l'homme, et des forces qui peuvent agir sur lui» ${ }^{18}$.

À cette partie critique suit, dans l'entretien, une partie positive où la question des unions libres est résolue par l'argument de la repopulation. Ici, la conversion progressive de l'Aumônier est scandée par deux astuces narratives. La première est une note de la main de ce dernier, retranchée du dialogue et découverte par A. Cette irruption, qui fait du religieux le transcripteur du dialogue, révèle l'authentique point de vue de ce personnage sur la question sexuelle. Encore une fois, le récit glisse de la fiction à la réalité du cadre:

A: Qu'est-ce que je vois là en marge?

B: C'est une note où le bon aumônier dit que les préceptes des parents sur le choix des garçons et des filles étaient pleins de bon sens et d'observations très fines et très utiles, mais qu'il a supprimé ce catéchisme qui aurait paru à des gens aussi corrompus et aussi superficiels que nous d'une licence impardonnable; ajoutant toutefois que ce n'était pas sans regret qu'il avait retranché des détails où l'on aurait vu premièrement jusqu'où une nation qui s'occupe sans cesse d'un objet important peut être conduite dans ses recherches sans les secours de la physique et de l'anatomie. Secondement, la différence des idées de la beauté dans une contrée où l'on rapporte les formes au plaisir d'un moment, et chez un peuple où elles sont appréciées d'après une utilité plus constante. [...] Il n'y a presque rien de commun entre la Vénus d'Athènes et celle d'Otaïti; l'une est Vénus galante, l'autre est Vénus féconde. (Supplément, pp. 561-562)

Cette technique permet à l'auteur de donner plus d'importance au commentaire omis, l'insérant dans un réseau polyphonique qui en ratifie le sens. Ainsi, comme dans une sorte de mise en abyme, c'est la voix de Diderot qu'on entend en écoutant B, lequel valide, par sa lecture, la pensée de l'Aumônier ${ }^{19}$. La seconde des astuces est un discours exemplaire, inséré entre la troisième et la quatrième section du Supplément. Il s'agit de l'épisode de Miss Polly Baker que l'auteur déclare avoir tiré de l'Histoire des deux Indes ${ }^{20}$. Devenue grosse pour la cinquième fois, la fille tient devant le tribunal un plaidoyer contre les lois injustes: dans la Nouvelle-Angleterre, «la loi condamne toutes les personnes du sexe qui ne doivent le titre de mère qu'au libertinage à une amende ou à une punition corporelle» (Supplément, p. 562). Ici comme plus haut, l'enchâssement des voix - c'est encore B qui reproduit un «discours second» - a la fonction de renforcer l'idée centrale, à savoir la critique du philosophe contre l'absurdité des opinions reçues.

D'ailleurs, les motifs qui ont déterminé Diderot à privilégier les arguments d'une morale utilitariste et sociale se devinent facilement: un des plus évidents est la réaction contre le célibat des prêtres et contre l'état monastique. L'«Entretien» en effet se termine par le triomphe de la nature, représenté, sur le plan de la communication, par les formes d'un dialogue novateur. Dépassant les modèles traditionnels, Diderot met le langage en contact avec le monde et réhabilite le corps comme vecteur sémantique

(18) G. Chinard, Introduction, dans D. Diderot, Supplément au Voyage de Bougainville, Paris-Baltimore, Droz-The Johns Hopkins Press, 1935, p. 69.

(19) «Sur le plan linguistique, l'accès aux pensées d'autrui est permis à tout locuteur qui utilise les modalités du discours rapporté ou transposé ou narrativisé» (A. RABATEL, La construction textuelle du point de vue, Lausanne-Paris, Delachaux \& Niestlé, 1998, p. 151).

(20) Cette histoire ne serait, à l'origine, qu'une mystification de Benjamin Franklin. Elle parut en France en 1770 dans la première édition de l'Histoire des deux Indes. Les ajouts qu'elle subit dans les éditions suivantes $(1774,1780)$ attestent l'intervention de Diderot et font écho au Supplément: le discours de cette fille devant ses juges serait souvent répété «dans nos contrées et partout où l'on attache des idées morales à des actions physiques qui n'en comportent pas, si les femmes y avaient l'intrépidité de Polly Baker» (G.-Th. Raynal, Histoire des deux Indes, VIII, XVII). La genèse de l'épisode, inséré dans la version finale du Supplément, est retracée par H. DiECKMANN (voir notamment les pages XXIV-XXVI de son édition). 
de l'expérience. La dernière réplique de l'Aumônier en est la preuve, car elle montre la vacuité de la parole face au travail des sens:

Le bon aumônier raconte qu'il passa le reste de la journée à parcourir l'île, à visiter les cabanes, et que le soir, après souper, le père et la mère l'ayant supplié de coucher avec la seconde de leurs filles, Palli s'était présentée dans le même déshabillé que Thia, et qu'il s'était écrié plusieurs fois pendant la nuit «Mais ma religion! mais mon état!», que la troisième nuit il avait été agité des mêmes remords avec Asto l'aînée, et que la quatrième, il l'avait accordée par honnêteté à la femme de son hôte. (Supplément, p. 571)

Hors du contexte européen, la «religion» et l'«état ecclésiastique» sont des mots inopérants. Audrey Guitton offre une bonne explication de cette démarche: «Le dialogue, en tant que langage verbal, est doublé d'un second dialogue corporel qui progresse plus rapidement que le premier et qui finit par le dépasser: les objections de l'aumônier paraissent de plus en plus dérisoires à la mesure que son corps accepte les préceptes que lui propose Tahiti» ${ }^{21}$.

Le reste du Supplément est occupé par la «Suite du dialogue entre A et B». À partir de ce moment, le ton rationnel du commentaire reprend sa primauté; les répliques multiplient les questions et les précautions oratoires, l'échange reproduit une pensée en gestation. Qu'est-ce qu'il faut conclure de la fiction tahitienne? «Quelles conséquences utiles à tirer des mœurs et des usages bizarres d'un peuple non civilisé?» (Supplément, p. 572). Au-delà du dilemme traditionnel (état de natureétat de société), c'est le problème d'une réforme à effectuer qui est soulevé dans ces pages. Loin d'épouser une voie unique, Diderot reprend les thèmes de l'«Entretien» pour les relire dans l'optique de l'homme moderne. C'est son idée de bonheur et, en conséquence, sa vision de l'être humain qui émergent ici, au fur et à mesure que l'on discute des problèmes moraux concrets: «en admettant que l'état de civilisation soit plein d'abus et que ces abus doivent être réformés sur le modèle de l'état ou de la loi de nature, il faut d'abord définir jusqu'à quel point les concepts ou les règles de la morale existante et certains sentiments qui leur sont liés sont dans la nature» ${ }^{22}$. À cette enquête correspond un dialogue de plus en plus complexe, où pullulent les conjonctions de restriction et d'opposition (si, mais...): A et B passent en revue le mariage, la galanterie, la coquetterie, la constance ou la fidélité, la jalousie et la pudeur. Mais leur discours tombe dans une impasse: «Vices et vertus - s'exclame B - tout est également dans la nature» (Supplément, p. 575); même chez les Tahitiens, il y a une bonne et une mauvaise nature. Ainsi, pour décrire la morale naturelle, il ne reste à l'auteur qu'à évoquer ce qu'elle n'est pas et à reprendre sa critique sociale ${ }^{23}$. Au point de vue philosophique, l'image de l'Autre se corrompt, perd son rôle de modèle, et fusionne avec

(21) A. Guitton, L'Autre lointain en dialogue. La quête de la voix idéale au siècle des Lumières, Paris, Classiques Garnier, 2012, p. 348.

(22) H. Dieckmann, Introduction, dans D. Diderot, Supplément au Voyage de Bougainville cit., p. LI.

(23) Ce changement de perspective se retrouve par exemple dans le discours que fait B sur l'origine de la dépravation sexuelle en France. Il résume en sept points les arguments avancés par Orou dans l'«Entretien»: 1 . «C'est par la tyrannie de l'homme qui a converti la possession de la femme en une propriété»; 2. «Par les mœurs et les usages qui ont surchargé de conditions l'union conjugale»; 3. «Par les lois civiles qui ont assujetti le mariage à une infinité de formalités»; 4. «Par la nature de notre société où la diversité des fortunes et des rangs a institué des convenances et des disconvenances»; 5. «Par une contradiction bizarre et commune à toutes les sociétés subsistantes où la naissance d'un enfant toujours regardée comme un accroissement de richesse pour la nation, est plus souvent et plus sûrement encore un accroissement d'indigence dans la famille»; 6. «Par les vues politiques des souverains qui ont tout rapporté à leur intérêt et à leur sécurité»; 7. «Par les institutions religieuses qui ont attaché les noms de vices et de vertus à des actions qui n'étaient susceptibles d'aucune moralité» (Supplément, p. 577). 
l'idée d'humanité: «Diderot comprend bien qu'à force de se rapprocher de l'autre, on finit par le faire disparaître en l'absorbant en soi. L'homme naturel cesse d'être une réalité pour devenir une vérité intérieure» ${ }^{24}$. C'est donc un homme multiple qu'il faut chercher, réunissant dans sa substance nature et sociétée $e^{25}$ :

B: Voulez-vous savoir l'histoire abrégée de presque toute notre misère? La voici. Il existait un homme naturel; on a introduit au-dedans de cet homme un homme artificiel, et il s'est élevé dans la caverne une guerre continuelle qui dure toute la vie. Tantôt l'homme naturel est le plus fort, tantôt il est terrassé par l'homme moral et artificiel; et dans l'un et l'autre cas le monstre est tiraillé, tenaillé, tourmenté, étendu sur la roue, sans cesse gémissant, sans cesse malheureux, soit qu'un faux enthousiasme de gloire le transporte et l'enivre, ou qu'une fausse ignominie le courbe et l'abatte. (Supplément, p. 578)

Vidé de toute identité, le Sauvage devient dorénavant un outil de connaissance de soi. On retrouve, dans ce recentrement, la pensée militante des Lumières ${ }^{26}: \ll \mathrm{Ab}$ sorbé par le spectacle de sa propre histoire, l'homme européen se détourne de tout ce qui n'est pas elle, et ne parvient à s'intéresser au monde sauvage que dans la mesure où celui-ci offre l'image de son passé, ou d'un présent encore enténébré» ${ }^{27}$. Il est frappant de constater à quel point la structure du Supplément suit ces basculements: d'une part, par la distribution des voix dans ses parties (dans le dialogue final l'Autre lointain n'est plus sur scène et le dernier mot est confié à l'Européen), d'autre part, par la valeur plastique du dialogisme (l'élocution douteuse témoigne d'une réflexion en acte et, par ses locuteurs, d'un modèle d'individu hétéroclite). Du reste, le rêve diderotien d'un «moyen terme» (tel qu'il l'exprime par exemple dans la Réfutation d'Helvétius) a échoué; aucun état intermédiaire n'est parvenu à fixer «l'homme civilisé entre l'enfance du sauvage et notre décrépitude» ${ }^{28}$.

C'est plutôt par la conscience de sa multiplicité que l'être humain peut accéder au bonheur. Diderot résume ce point dans une phrase devenue proverbiale: incapables d'adhérer à un seul modèle ${ }^{29}$, «imitons le bon aumônier, moine en France, sauvage dans Otaïti» (Supplément, p. 581). La même solution relativiste est proposée aux niveaux social et politique:

A: Prendre le froc du pays où l'on va, et garder celui du pays où l'on est. (Supplément, p. 581)

S'éclaire par là le projet philosophique de l'Histoire des deux Indes ${ }^{30}$, mais aussi de la Réfutation d'Helvétius, où le thème de la «diversité» revient au premier plan.

(24) A. Ben Ali, Diderot et Bougainville: interpréter ou décrire l'altérité, dans Journées Diderot, Actes du Colloque organisé par le Département de Français (9-10 novembre 1984), Tunis, Publications de la Faculté des Lettres de la Manouba, 1990, p. 117.

(25) On pourrait voir dans ce mariage le projet de L'Ingénu. Voir, entre autres, L. Sozzi, Un selvaggio a Parigi. Miraggio utopico e progetto politico nel "Viaggio attorno al mondo" di Bougainville e nel "Supplemento" di Diderot, Roma, Edizioni di Storia e Letteratura, 2009, p. 225.

(26) Pensons aux contes de Voltaire, mais aussi à ses Entretiens d'un sauvage et d'un bachelier (1761).

(27) M. Duchet, Anthropologie et histoire au siècle des Lumières, Paris, Albin Michel, 1995, p. 12.

(28) D. DiDERot, Réfutation d'Helvétius, dans CEuvres philosophiques cit., p. 614. Quant à ce projet, le penseur ne tardera pas à avouer ses doutes: «Dans tous les siècles à venir, l'homme sauvage s'avancera pas à pas vers l'homme civilisé. L'homme civilisé reviendra vers son état primitif, d'où le philosophe conclura qu'il existe dans l'intervalle qui les sépare un point où réside la félicité de l'espèce. Mais qui est-ce qui fixera ce point? Et s'il était fixé, quelle serait l'autorité capable d'y diriger, d'y arrêter l'homme?» (Histoire des deux Indes, IV, IX).

(29) «A: Ainsi vous préféreriez l'état de nature brute et sauvage? - B: Ma foi, je n'oserais prononcer» (Supplément, p. 579).

(30) Le titre complet l'affiche d'ailleurs comme une «histoire philosophique et politique». Voir surtout l'Introduction de la troisième édition (1780), où Diderot nous livre son testament intellectuel. 
C'est l'histoire d'une division interne à l'homme que trace, au terme de ce parcours, l'agencement des dialogues en alternance. De par sa complexité, la structure du Supplément donne un juste aperçu des débats qui le traversent. Texte polyphonique, il accumule les contrastes et les ruptures pour mieux afficher une pensée en train de se re-faire. Le montage des discours est en ce sens crucial: démultiplier les regards revient, chez Diderot, à adopter une attitude épistémologique qui épouse les manifestations mêmes de l'humanité, comme la seule possibilité de l'appréhender dans ses multiples facettes. Ce qui est en jeu, dès lors, n'est ni un système ni une thèse définitive, mais un questionnement qui permet de débusquer les réseaux idéologiques sous-jacents.

Plusieurs procédés attestent cette pluralisation. D'abord, la pratique dialogique, qui fait fonction, chez l'auteur, d'exercice herméneutique. Le Supplément, on l'a vu, échappe à la tradition platonicienne: «La forme dialoguée, ici, ne repose pas sur l'opposition traditionnelle de la thèse et de l'antithèse, mais au contraire sur la réversibilité toujours possible des points de vues ${ }^{31}$. Ce qui permet de mettre en scène une réalité changeante, décrite à partir d'une série de concepts clés. Parmi ceux-ci, les notions d'identité et d'altérité jouent un rôle fondamental. Si d'une part elles servent de pivot à l'imaginaire primitiviste (la rencontre de deux cultures pose le problème de l'attitude envers l'étranger), de l'autre elles sous-tendent une réflexion sur l'homme, que les pages du Supplément tentent d'articuler. Les actes de parole, les voix tantôt plus fortes tantôt plus faibles des locuteurs, sont orchestrés, dès lors, de façon à traduire les différentes phases de la pensée auctoriale. La longueur des répliques (leur style dubitatif) témoigne de cette correspondance, reflétée, de surcroît, par un dialogue de moins en moins verbal. L'introduction du corps comme vecteur sémantique du récit est l'une des nouveautés de ce dialogue, doté par Diderot d'une fonction mimétique.

C'est dans l'effort de peindre la réalité, la rencontre du Moi français et de l'Autre tahitien, que la structure du Supplément se complexifie. Les plans narratifs s'entremêlent, la parole du Sauvage est tantôt modifiée (traduite, rapportée), tantôt soutenue par des voix secondaires ${ }^{32}$. C'est une nouvelle affirmation identitaire, une contrenarration de l'homme, qui se dessine ici à travers une dialectique signifiante: l'altérité est indissociable de l'ipséité; le soi et l'autre deviennent corrélatifs ${ }^{33}$.

L'originalité de Diderot tient sans doute à cette configuration, anticipée par le dialogue corporel de l'Aumônier et incarnée, à la fin du texte, par les répliques de B. Dans son portrait des mœurs tahitiennes, le philosophe donne au lecteur les moyens émotionnels de se connaître, tout en étendant ses intuitions aux thèmes fondamentaux de son ouvrage: «L'exigence morale et le sens de l'urgence politique vont de pair chez Diderot avec la revendication de Tahitis intimes» ${ }^{34}$.

Revendication que l'écriture plurielle du Supplément relie sans cesse aux multiples présents de l'Histoire ${ }^{35}$.

PIERINO GALLO

IHRIM, Saint-Étienne

(31) S. Pujol, Le Dialogue d'idées au dix-buitième siècle cit., p. 308.

(32) G. DANIEL place ces phénomènes à l'origine du «dialogisme métaleptique» de Diderot (voir Le Style de Diderot. Légende et structure, Genève-Paris, Droz, 1986, pp. 393-394).

(33) «Nous avons respecté notre image en toi» s'écriait le vieillard (Supplément, p. 548).

(34) M. Delon, Préface, dans D. Diderot, Supplément au Voyage de Bougainville, Paris, Gallimard, 2002, «Folio Classique», p. 25

(35) Une première version, inédite, de ce texte fut présentée lors du colloque «La scène de la création chez Diderot» (Institut Supérieur des Sciences Humaines de Tunis, 5-6 mars 2015). 